\title{
Application of a bone conserving revision stem for unstable intertrochanteric fractures in senile osteoporotic population
}

MENGCUN CHEN ( $\square$ chenmc0603@163.com )

Wuhan Union Hospital https://orcid.org/0000-0002-1060-644X

Jinlong Wang

Wuhan Union Hospital

Shuhua Yang

Wuhan Union Hospital

Xianzhe Liu

Wuhan Union Hospital

\section{Research Article}

Keywords: Hip, Unstable intertrochanteric fracture, Osteoporosis, Bipolar hemiarthroplasty, cementless stem, fully hydroxyapatite coating

Posted Date: April 19th, 2021

DOI: https://doi.org/10.21203/rs.3.rs-409922/v1

License: (c) (i) This work is licensed under a Creative Commons Attribution 4.0 International License. Read Full License 


\section{Abstract \\ Purpose}

Primary hemi-arthroplasty is gaining rising attention to treat unstable intertrochanteric fractures in senile patients with severe osteoporosis. The objective of this study is to evaluate the early clinical and radiographic outcomes using bone conserving revision stem for unstable intertrochanteric fractures in senile osteoporotic population.

\section{Methods}

A retrospective observation of a series of 31 patients with unstable intertrochanteric fractures were conducted. The average age of the patients enrolled was 82.1 years and all patients underwent primary hemi-arthroplasty with the application of bone conserving and fully porous-coated revision stem. Clinical and radiographic evaluations during both the hospital-stay and follow-up were performed.

\section{Results}

The 31 patients were followed up for an average of 23 months postoperatively. The average operation time was 74.2 min while the mean intraoperative blood loss was $200.1 \mathrm{ml}$, with an average $11.1 \mathrm{~g} / \mathrm{L}$ decrease of hemoglobulin after the procedure. The mean Harris hip score was 82.1 and visual analog scale was 1.7 at the latest follow-up. No intra-operative or postoperative peri-prosthetic fracture was noted. Postoperative complications included one thrombosis formation in posterior tibial vein and one congestive heart failure, both patients were discharged uneventfully after treatment. Twenty-one (21/31, $67.7 \%)$ patients regained their pre-injury walking activities. Radiographically, none of these hips had evident loosening of the stem or osteolysis. With follow-up period of 23 months, the mortality rate was $3.2 \%(1 / 31)$ with no revision required.

\section{Conclusion}

Primary hemi-arthroplasty with application of the bone conserving, cementless revision stem could serve as a reliable alternation in treatment of unstable intertrochanteric fractures in senile population with osteoporosis.

\section{Introduction}

Hip fractures are common in our clinical practice, with 1.6 million cases annually worldwide and a predicted worldwide occurrence of 6.3 million by 2050 with extended life expectancy(1). Surgical management for intertrochanteric fracture in the geriatric population remains challenging due to their medical comorbidity, poor bone quality and fracture instability(2). Mostly, osteosynthesis with internal 
fixation is the most commonly used for stable intertrochanteric fractures and leads to satisfactory clinical outcomes(3). However, the application of internal fixation has been debated under circumstances related to instability of fracture and severe osteoporosis $(4,5)$. Complications following the internal fixation of intertrochanteric fractures are nonunion, malunion, fracture collapse with intra-articular screw migration and post-traumatic osteoarthritis, which would result in significant loss of mobility and increase considerable burden of medical sources(4-6).

Joint arthroplasty is unanimously considered as a salvage option for failed internal fixation $(2,4)$. However, the conversion procedure could be difficult for many reasons(4). Besides, the senile population was usually at risk of not only cardiovascular, pulmonary, and neurological co-morbidities which could diminish the opportunity for a second operation(2), but also the psychological and economic burden brought by the additional procedure. Recently, there were rising trends to treat unstable intertrochanteric fractures in senile patients with primary hemi-arthroplasty with encouraging early and mid-term clinical outcomes(7-9). However, joint arthroplasty was usually accompanied by prolonged surgical time and increased intra-operative blood loss(2). Additionally, poor stem press-fit and biological fixation failure were also concerned in the absence of proximal femoral stability due to fracture and osteoporosis(10). It would be crucial to determine an optimized surgical and rehabilitation protocol individually after comprehensive pre-operative assessment.

A cementless, bone conserving and fully-hydroxyapatite coated femoral revision stem has been widely utilized in hip revision procedures(11). The simplistic surgical techniques for femoral stem implantation could minimize iatrogenic bone loss and reduce surgical time(12). The initial stability could be achieved at the metaphyseal junction and the fully coated hydroxyapatite could potentially provide an optimal osseointegration surface and obtain long-term stability $(12,13)$, which could be suitable in unstable intertrochanteric fractures in senile patients with osteoporosis $(7,8)$. However, there were few research papers illustrating the application of the current revision femoral stem in a senile, osteoporotic and unstable intertrochanteric fracture(7). Therefore, the objective of the current study was to evaluate the early clinical and radiographic outcomes by using the revision femoral stem in primary hemi-arthroplasty for treatment of unstable intertrochanteric fractures in senile osteoporotic population.

\section{Patients And Methods}

\section{Patient demographics}

From July 2017 to April 2018, 31 patients (31 hips) were retrospectively reviewed in the present study. All the patients admitted for intertrochanteric fractures would receive a comprehensive appraisal by a Multiple Disciplinary Team (MDT) consists of a trauma surgeon, a joint surgeon, a physician of intensive care unit, an anesthesiologist and a physical therapist. The indications for a primary hemi-arthroplasty were: 1. Patients who have sustained an unstable intertrochanteric fracture (three parts or more intertrochanteric fractures with a loss of posteromedial cortical buttress); 2 . Patients who are at the age of 75 years or older at the time of injury; 3 . Patients who were able to ambulate independently with or 
without crutches (Koval's grade I-V)(14), and 4. Patients who were assigned as class I-III by American Society of Anesthesiologist (ASA) score(15). Preoperative demographic data including age at the time of the surgery, gender, body mass index (BMI), comorbidities, types of fracture, American Society of Anesthesiologist score, and pre-fracture ambulatory status by Koval's categories were obtained by reviewing medical records and was listed in Table 1. 
Table 1

Demographics of patients underwent primary hemiarthroplasty

\begin{tabular}{|ll|}
\hline Demographic & \\
\hline Number of patients & 31 \\
\hline Gender & \\
\hline Male & 14 \\
\hline Female & 17 \\
\hline Average age (years) & $82.1(75-92)$ \\
\hline Comorbidity & \\
\hline Hypertension & $20(64 \%)$ \\
\hline Diabetes & $10(32 \%)$ \\
\hline Cerebrovascular accident & $4(13 \%)$ \\
\hline COPD & $3(10 \%)$ \\
\hline Auricular fibrillation & $3(10 \%)$ \\
\hline Coronary heart disease & $2(6 \%)$ \\
\hline Parkinson's disease & $1(3 \%)$ \\
\hline Chronic renal failure & $1(3 \%)$ \\
\hline Evans-Jesen classification & 12 \\
\hline III & 7 \\
\hline IV & 11 \\
\hline V & 13 \\
\hline ASA class & 11 \\
\hline I II & 19 \\
\hline II & \\
\hline II & 11 \\
\hline
\end{tabular}




\section{Demographic

\begin{tabular}{|ll|}
\hline IV & 5 \\
\hline ASA, American Society of Anesthesiologist & 3 \\
\hline
\end{tabular} \\ Implant characteristics}

A cementless, tapered titanium femoral stem with extensive hydroxyapatite coating on the surface was used in the hemi-arthroplasty procedure. The design was based on the CORAIL and KAR ${ }^{\text {TM }}$ Hip Systems (Depuy, DePuy International). Theoretically, the extended length of the stem could achieve initial stability with fractured proximal femur at metaphyseal level and the fully coated hydroxyapatite could potentially provide an optimal osseointegration surface. The stem has also been engineered to transfer maximum load to the remaining bone in the proximal femur to aid in reconstitution and stability. Additionally, the less aggressive but more simplified surgical technique of the stem could potentially preserve the bone stock during implantation (Fig. 1). The acetabular component is a corresponding cobalt-chromium cup with an ultra-high molecular weight polyethylene liner inside. A 28-mm metal head was used in all hips.

\section{Anesthesia and surgery}

The anesthesia was determined by the MDT team after a comprehensive consultation and appraisal of the patient. Spinal anesthesia was preferred to minimize postoperative delirium(3). General anesthesia was selected as an alternative for patients with contraindications of spinal anesthesia (such as thrombocytopenia, abnormal coagulation function, lumbar spondylolisthesis, et al.). A total of 25 patients were operated under spinal anesthesia, and 6 under general anesthesia in the present study.

All operations were performed through a minimal invasive posterolateral approach by the same senior joint surgeon. The greater and lesser trochanters were fractured off the main fragment of femur in all cases involved in the present study. To adjust lower limb length, we modified the method described by Lee et al(7), the two poles of the patella were palpated instead of the tibial tuberosity (Fig. 2). With the use of the trial components, the optimal stem size and head length were determined. If the size, stability, and leg length were satisfactory, the stem was inserted into the femoral canal with a firm impaction from a gentle tapping to a press fit. Then, the greater trochanteric and the medial fracture fragments were reattached and fixed with two to three titanium cables. A Greater Trochanteric Reattachment Plate (Zimmer Biomet Corporation, USA) was utilized if necessary. No drainage tube was placed in any of the patients

\section{Postoperative care}

Patients were instructed to stand with assistance, with weight-bearing as tolerated and a walking aid on postoperative day 1 (POD1). The rehabilitation protocol was designed based on the progress of the 
walking ability of the patient by a physical therapist. Pharmacological prophylaxis for venous thromboembolism was performed routinely in all the patients

\section{Data collection}

The operation time, intraoperative blood loss, iatrogenic fractures, length of hospitalization, and blood transfusion were noted during the hospital-stay. Postoperative complications such as dislocations, deep venous thrombosis, infections, peri-prosthetic fractures and frontal thigh pain were also recorded.

Follow-up evaluations were performed at 1, 3, 6, and 12 months and annually thereafter in the outpatient clinic. For clinical evaluations, Koval's categories (14)was used for activity level and Harris hip score (HHS) (16)was used for functional assessment. Activity levels were defined as follows: level I, independent community ambulator; level II, community ambulator with cane; level III, community ambulator with walker/crutches; level IV, independent household ambulator; level V, household ambulator with cane; level VI, household ambulator with walker/crutches; and level VII, nonfunctional ambulatory(14). The pain was assessed using visual analog scale (VAS) that was grade as no pain (0-2 points), mild (3-5 points), moderate ( $6-8$ points), and severe ( $>8$ points). Radiographically illustrations in terms of the osteolysis, bone in-growth(17), subsidence of the femoral components, lower limb discrepancy and heterotopic ossification were collected at each follow-up. All the clinical and radiographic information was collected by two independent research assistants.

\section{Statistical Analysis}

SPSS software (version 23.0; IBM) was used for statistical analysis of primary data. Categorical variables are presented as the median and interquartile range or as the number and percentage.

\section{Results}

The 31 patients were followed up for 14-26 months, with an average of 23 months. One patient died at 15 months after the operation due to respiratory failure. The patients' records during the hospital-stay were indicated in Table 2. The mean operation time was (74.2 \pm 12.1$)$ min (range, 62-95 min). The mean intraoperative blood loss was $(200.1 \pm 70.2) \mathrm{mL}$ (range, $150-400 \mathrm{~mL}$ ). The mean hemoglobulin $(\mathrm{Hb})$ on POD1 was (82.1 \pm 11.2$) \mathrm{g} / \mathrm{L}$ (range, $70-109 \mathrm{~g} / \mathrm{L}$ ), with an average decrease of $(11.1 \pm 6.0) \mathrm{g} / \mathrm{L}$ (range, 6$25 \mathrm{~g} / \mathrm{L}$ ) postoperatively. The mean length of stay was (14.3 \pm 3.3 ) days (range, 10-21 days). Of the 31 patients, $3(9.7 \%)$ required transfusion with the average transfusion of $2.0 \pm 0.7$ units (range, 1.5-3 units). No iatrogenic fracture was noted during the operation. 
Table 2

In-hospital record of patient underwent primary hemi-arthroplasty

\begin{tabular}{|ll|}
\hline In-hospital Record & \\
\hline Intraoperative blood loss $(\mathrm{mL})$ & $200.1 \pm 70.2$ (range, 150-400) \\
\hline Hb of POD1 (g/L) & $82.1 \pm 11.2$ (range, 70-109) \\
\hline Length of stay (days) & $14.3 \pm 3.3$ (range, 10-21) \\
\hline Blood transfusion & $3(9.7 \%)$ \\
\hline Number (\%) & $2.0 \pm 0.7$ (range, 1.5-3) \\
\hline Volume (units) & \\
\hline Hb: Hemoglobulin; POD1: Postoperative Day 1 \\
\hline
\end{tabular}

Postoperative complications were noted in 2 cases $(2 / 31,6.5 \%)$ before discharge. Thrombosis in posterior tibial vein was noted in one patient, he was discharged after placement of a lower extremity venous filter. Another patient had coronary heart disease and atrial fibrillation before surgery and was found to have congestive heart failure on POD4, she was discharged after cardiovascular medical treatment (Spironolactone $40 \mathrm{mg}$ po Bid, Bisoprolol fumarate $5 \mathrm{mg}$ po qd, aspirin $100 \mathrm{mg}$ po qd) from the cardiovascular department. All the patients could ambulate independently with a walker before discharge.

The VAS score was $(2.4 \pm 0.8)$ at 4 weeks and was $(1.7 \pm 0.7)$ at 1 year postoperatively, which was significantly lower than the pre-operative value $(5.4 \pm 0.9, p<0.05)$. The mean HHS was $(82.1 \pm 4.8)$ at 1 year after the procedure. According to Koval's categories, 21 patients $(21 / 31,67.7 \%)$ regained pre-injury ambulatory status, $7(7 / 31,22.6 \%)$ patients dropped 1 level of ambulatory ability, 3 patients $(3 / 31,9.7 \%)$ who ambulated independently without crutches before injury, but they require a walker for community ambulation at the latest follow-up. Four (12.9\%) out of 31 patients had the limb length discrepancy with a range of 3-7 mm without clinical manifestations. No postoperative complications were observed in any of the patients during the follow-up.

Radiographically, all femoral stems showed radiographic evidence of bone ingrown stability at final follow-up(17). Periprosthetic osteolysis and aseptic loosening were not detected around the stem. None of these patients had evidence of subsidence of femoral stem $\geq 5 \mathrm{~mm}$. (Fig. 3)

\section{Discussion}

In this retrospective study we evaluated the early clinical and radiographic outcomes for the application of cementless, bone conserving and fully hydroxyapatite coated revision stem in primary hemiarthroplasty for unstable intertrochanteric fractures. The treatment outcomes at average 23-monthfollow-up were encouraging. Clinically, patients gained immediate pain relief with early mobilization and prompt return to pre-fracture activity levels with an acceptable mortality rate. Radiographically, stem 
stability and implant bone ingrowth were achieved at the final follow-up without evident subsidence of femoral stem. Taken together, the results of the current study demonstrated that the current stem played an important role in the treatment of unstable intertrochanteric fractures, especially in senile patients with osteoporosis.

Primary joint arthroplasty acquired arising attention in the treatment of unstable intertrochanteric fractures in elderly patients with osteoporosis. Compared to internal fixation, concerns of joint arthroplasty persisted in prolonged surgical time and increased intra-operative blood loss, which could be lethal to geriatric patients with co-morbidities(2). However, in the present study, the average surgery time could be limited to $(74.2 \pm 12.1) \mathrm{min}$, which is shorter than the time reported in the literature for joint arthroplasty $(7,8)$, and was comparable with the time consumed of an internal fixation procedure for complicated intertrochanteric fractures(18). Additionally, a recent meta-analysis indicated that there was no significant difference between the hip replacement and intramedullary nail groups in terms of length of surgery(18). Although many investigators reported increased intra-operative blood loss in joint arthroplasty compared to intramedullary nail fixation, the rational application of tranexamic acid contributed to the limited blood loss in the present study. The average intra-operative blood loss was $(200.1 \pm 70.2) \mathrm{ml}$ and the average decrease of $\mathrm{Hb}$ was $(11.1 \pm 6.0) \mathrm{g} / \mathrm{L}$, with only $3(9.7 \%, 3 / 31)$ patients transfused postoperatively. Although recent meta-analysis reported a statistically increased intraoperative blood loss in hemi-arthroplasty, the authors admitted that the results possessed obvious heterogeneity and the difference might be derived from different devices and levels of expertise of surgeons(18). With minimal invasive posterolateral approach, simplistic techniques for stem implantation and coordination of the MDT group, the effects brought by the operation and anesthesia could be minimized in the present study.

The arthroplasty could shorten the time to immobilize and allowed the patients to full weight bearing as soon as possible postoperatively, which had a notable effect on reducing mortality in hip fractures and preventing complications resulted from long-term bedridden $(18,19)$. Haentjens et al. found a higher incidence of pneumonia and pressure sores with internal fixation due to restricting early weight-bear mobilization(20). While losifidis et al reported that early walking ability after hip fracture of elderly patients was the most significant predictive factor in their long-term survival study with 230 patients(21). In the present study, patients were approved to mobilize one day after the surgery with weight-bearing as tolerated. The rapid pain relief and early stabilization of the joint dramatically simplified the postoperative nursery process and enhanced the rehabilitation progression. Significantly improved VAS and HHS with few incidences of postoperative complications were observed during the hospital-stay, while nearly $70 \%$ of the patients returned to their pre-injury activity level at the final follow-up. Additionally, the mortality rate was as low as $3.2 \%$ (1/31) during the23 months follow-up, which was much lower than the rate reported in the previous literature. Indeed, these superior results might be attributed to the limited duration of our observation and the small number of patients involved. Nevertheless, we could also postulate that the results were related to the early mobilization and prompt return to pre-fracture activity level introduced by the peri-operative MDT caring protocol. 
It was reported that the failure of osteosynthesis or re-operation rate of internal fixation could range from 6-32\% for elderly patients with fracture instability, comminution, and osteoporosis, which could exhaust considerable medical sources(6). However, successful bony in-growth was achieved in all patients with no evident implant loosening or subsidence in the present study. The revision stem used in the current study was designed to fill the meta-diaphyseal junction and could obtain long-term biologic fixation with proximal bone loss(22). The extensive hydroxyapatite coating, which was proven to increase biological fixation and allows for even stress distribution and good long-term survival, could also enhance stability via fixation through the bone-hydroxyapatite interface $(12,13)$. Additionally, guaranteed initial stability and early weight-bearing protocol would also augment osteointegration of the fracture(23). Although long-term follow up might witness a higher rate of implant loosening or revision, the clinical relevance could be debilitated due to lower activity level and limited life expectancy in the elderly population.

The application of the current stem provided a less aggressive but more simplified option in treatment of unstable intertrochanteric fractures in the senile osteoporotic population. A typical revision stem enabled the surgeon to engage the diaphysis to obtain stability distally(24). However, these conventional revision stems require aggressive reaming out to the host cortical bone to properly size the femoral component and prevent subsidence, which could cause frontal thigh pain and proximal stress shielding in $10-24 \%$ of cases $(11,25)$. However, no complaint of thigh pain was observed during the follow-up in the current study. The more proximal press-fitting at the metaphyseal junction of the femoral stem may deliver a more friendly bone loading and stress distribution. Furthermore, the proprietary slots quadrants incorporated in the distal portion of the stem were designed to provide adaptability to the natural curve of the femur. Additionally, in the absence of the diaphyseal reaming, which was reported to be associated with high rate of peri-operative femoral fracture(11), the iatrogenic bone loss could be minimized. No iatrogenic fracture was observed intra-operatively and no peri-prosthetic fracture was noted till the latest follow-up.

The current study is not without limitations. It was a small and short retrospective observation, and the influence of subsidence and potential loosening of the implant requires assessment over a longer period. However, a long-term follow-up study is barely possible and has little clinical relevance in elderly patients, who have a short life expectancy and limited activity during their life. The absence of a control group would inevitably compromise the interpretation of the current results. The patient series involved in the presented study was highly selective, each patient was assessed by a group of MDT professionals to receive a primary hemi-arthroplasty instead of an internal fixation procedure, hence a comparison group could not be recruited.

\section{Conclusion}

Primary hemi-arthroplasty with the application of a bone conserving, cementless fully hydroxyapatitecoated revision stem could serve as a reliable alternation in treatment of unstable intertrochanteric fractures in senile population with moderate or evident osteoporosis. However, comprehensive pre- 
operative assessment, individualized peri-operative administration and coordination of both trauma and joint surgeons would be indispensable to guarantee a favorable treatment outcome.

\section{Declarations}

\section{Acknowledgements}

Not applicable.

\section{Abbreviations}

MDT Multiple Disciplinary Team

ASA American Society of Anesthesiologist

BMI body mass index

POD Postoperative day

HHS Harris hip score

VAS Visual analog scale

\section{Authors' contributions}

M.C. and J.W. were involved in data collection and follow-up assessments. S.Y. and X.L. were responsible for literature search and study design. M.C. and J.W. were responsible for drafting the manuscript. S.Y. and X.L. finalized the manuscript. All authors have read and approved the submitted manuscript.

\section{Funding}

Not applicable.

\section{Availability of data and materials}

The datasets used and/or analyzed during the current study are available from the corresponding author on reasonable request.

\section{Ethics approval and consent to participate}

The Ethics Committee of Tongji Medical College, Huazhong University of Science and Technology gave a final approval for this study. Although the data were collected anonymized and centrally, all patients signed written informed consent for participate.

\section{Consent for publication}


All patients signed written informed consent for publication.

\section{Competing interests}

The authors declare that they have no competing interests

\section{References}

1. Goodman SM, Springer B, Guyatt G, Abdel MP, Dasa V, George M, Gewurz-Singer O, Giles JT, Johnson B, Lee S, Mandl LA, Mont MA, Sculco P, Sporer S, Stryker L, Turgunbaev M, Brause B, Chen AF, Gililland J, Goodman M, Hurley-Rosenblatt A, Kirou K, Losina E, MacKenzie R, Michaud K, Mikuls T, Russell L, Sah A, Miller AS, Singh JA, Yates A. 2017 American College of Rheumatology/American Association of Hip and Knee Surgeons Guideline for the Perioperative Management of Antirheumatic Medication in Patients With Rheumatic Diseases Undergoing Elective Total Hip or Total Knee Arthroplasty. J Arthroplasty. 2017;32(9):2628-2638.

2. Socci AR, Casemyr NE, Leslie MP, Baumgaertner MR. Implant options for the treatment of intertrochanteric fractures of the hip: rationale, evidence, and recommendations. Bone Joint J. 2017;99-B(1):128-133.

3. Brox WT, Roberts KC, Taksali S, Wright DG, Wixted JJ, Tubb CC, Patt JC, Templeton KJ, Dickman E, Adler RA, Macaulay WB, Jackman JM, Annaswamy T, Adelman AM, Hawthorne CG, Olson SA, Mendelson DA, LeBoff MS, Camacho PA, Jevsevar D, Shea KG, Bozic KJ, Shaffer W, Cummins D, Murray JN, Donnelly P, Shores P, Woznica A, Martinez Y, Boone C, Gross L, Sevarino K. The American Academy of Orthopaedic Surgeons Evidence-Based Guideline on Management of Hip Fractures in the Elderly. J Bone Joint Surg Am. 2015;97(14):1196-1199.

4. Lee YK, Kim JT, Alkitaini AA, Kim KC, Ha YC, Koo KH. Conversion Hip Arthroplasty in Failed Fixation of Intertrochanteric Fracture: A Propensity Score Matching Study. J Arthroplasty. 2017;32(5):15931598.

5. Kashigar A, Vincent A, Gunton MJ, Backstein D, Safir O, Kuzyk PR. Predictors of failure for cephalomedullary nailing of proximal femoral fractures. Bone Joint J. 2014;96-B(8):1029-1034.

6. Makinen TJ, Gunton M, Fichman SG, Kashigar A, Safir O, Kuzyk PR. Arthroplasty for Pertrochanteric Hip Fractures. Orthop Clin North Am. 2015;46(4):433-444.

7. Lee YK, Ha YC, Chang BK, Kim KC, Kim TY, Koo KH. Cementless bipolar hemiarthroplasty using a hydroxyapatite-coated long stem for osteoporotic unstable intertrochanteric fractures. J Arthroplasty. 2011;26(4):626-632.

8. Kim JT, Kim HH, Kim JH, Kwak YH, Chang EC, Ha YC. Mid-Term Survivals After Cementless Bipolar Hemiarthroplasty for Unstable Intertrochanteric Fractures in Elderly Patients. J Arthroplasty. 2018;33(3):777-782.

9. Zha GC, Liu J, Wang Y, Feng S, Chen XY, Guo KJ, Sun JY. Cementless distal fixation modular stem without reconstruction of femoral calcar for unstable intertrochanteric fracture in patients aged 75 
years or more. Orthop Traumatol Surg Res. 2019;105(1):35-39.

10. Camurcu Y, Cobden A, Sofu H, Saklavc N, Kis M. What Are the Determinants of Mortality After Cemented Bipolar Hemiarthroplasty for Unstable Intertrochanteric Fractures in Elderly Patients? The Journal of arthroplasty. 2017;32(10):3038-3043.

11. Pinaroli A, Lavoie F, Cartillier JC, Neyret P, Selmi TA. Conservative femoral stem revision: avoiding therapeutic escalation. J Arthroplasty. 2009;24(3):365-373.

12. Makani A, Kim TW, Kamath AF, Garino JP, Lee GC. Outcomes of long tapered hydroxyapatite-coated stems in revision total hip arthroplasty. J Arthroplasty. 2014;29(4):827-830.

13. Reikeras O. Femoral revision surgery using a fully hydroxyapatite-coated stem: a cohort study of twenty two to twenty seven years. Int Orthop. 2017;41(2):271-275.

14. Christovam AC, Theodoro V, Mendonca FAS, Esquisatto MAM, Dos Santos GMT, do Amaral MEC. Activators of SIRT1 in wound repair: an animal model study. Arch Dermatol Res. 2019;311(3):193201.

15. Li C, Wang L, Zheng L, Zhan X, Xu B, Jiang J, Wu C. SIRT1 expression is associated with poor prognosis of lung adenocarcinoma. Onco Targets Ther. 2015;8:977-984.

16. Hu Q, Wu D, Ma F, Yang S, Tan B, Xin H, Gu X, Chen X, Chen S, Mao Y, Zhu YZ. Novel Angiogenic Activity and Molecular Mechanisms of ZYZ-803, a Slow-Releasing Hydrogen Sulfide-Nitric Oxide Hybrid Molecule. Antioxid Redox Signal. 2016;25(8):498-514.

17. Zheng XW, Shan CS, Xu QQ, Wang Y, Shi YH, Wang Y, Zheng GQ. Buyang Huanwu Decoction Targets SIRT1/VEGF Pathway to Promote Angiogenesis After Cerebral Ischemia/Reperfusion Injury. Front Neurosci. 2018;12:911.

18. Ju JB, Zhang PX, Jiang BG. Hip Replacement as Alternative to Intramedullary Nail in Elderly Patients with Unstable Intertrochanteric Fracture: A Systematic Review and Meta-Analysis. Orthop Surg. 2019;11(5):745-754.

19. Bonnevialle P, Saragaglia D, Ehlinger M, Tonetti J, Maisse N, Adam P, Le Gall C, French H, Knee S, Trauma Surgery A. Trochanteric locking nail versus arthroplasty in unstable intertrochanteric fracture in patients aged over 75 years. Orthop Traumatol Surg Res. 2011;97(6 Suppl):S95-100.

20. Das A, Huang GX, Bonkowski MS, Longchamp A, Li C, Schultz MB, Kim LJ, Osborne B, Joshi S, Lu Y, Trevino-Villarreal JH, Kang MJ, Hung TT, Lee B, Williams EO, Igarashi M, Mitchell JR, Wu LE, Turner N, Arany Z, Guarente L, Sinclair DA. Impairment of an Endothelial NAD(+)-H2S Signaling Network Is a Reversible Cause of Vascular Aging. Cell. 2018;173(1):74-89 e20.

21. Poulsen RC, Watts AC, Murphy RJ, Snelling SJ, Carr AJ, Hulley PA. Glucocorticoids induce senescence in primary human tenocytes by inhibition of sirtuin 1 and activation of the p53/p21 pathway: in vivo and in vitro evidence. Ann Rheum Dis. 2014;73(7):1405-1413.

22. Busch F, Mobasheri A, Shayan P, Lueders C, Stahlmann R, Shakibaei M. Resveratrol modulates interleukin-1beta-induced phosphatidylinositol 3-kinase and nuclear factor kappaB signaling pathways in human tenocytes. J Biol Chem. 2012;287(45):38050-38063. 
23. Zhang P, He L, Zhang J, Mei X, Zhang Y, Tian H, Chen Z. Preparation of novel berberine nano-colloids for improving wound healing of diabetic rats by acting Sirt1/NF-kappaB pathway. Colloids Surf B Biointerfaces. 2019:110647.

24. Chu X, Liu F, Huang J, Chen L, Li J, Tong P. Good short-term outcome of arthroplasty with Wagner SL implants for unstable intertrochanteric osteoporotic fractures. J Arthroplasty. 2014;29(3):605-608.

25. Rodriguez J, Deshmukh A, Robinson J, Cornell C, Rasquinha V, Ranawat A, Ranawat C. Reproducible fixation with a tapered, fluted, modular, titanium stem in revision hip arthroplasty at 8-15 years follow-up. The Journal of arthroplasty. 2014;29:214-218.

\section{Tables}

Table 1 Demographics of patients underwent primary hemi-arthroplasty

\begin{tabular}{|c|c|}
\hline \multicolumn{2}{|l|}{ Demographic } \\
\hline Number of patients & 31 \\
\hline \multicolumn{2}{|l|}{ Gender } \\
\hline Male & 14 \\
\hline Female & 17 \\
\hline Average age (years) & $82.1(75-92)$ \\
\hline \multicolumn{2}{|l|}{ Comorbidity } \\
\hline Hypertension & $20(64 \%)$ \\
\hline Diabetes & $10(32 \%)$ \\
\hline Cerebrovascular accident & $4(13 \%)$ \\
\hline COPD & $3(10 \%)$ \\
\hline Auricular fibrillation & $3(10 \%)$ \\
\hline Coronary heart disease & $2(6 \%)$ \\
\hline Parkinson's disease & $1(3 \%)$ \\
\hline Chronic renal failure & $1(3 \%)$ \\
\hline \multicolumn{2}{|l|}{ Evans-Jesen classification } \\
\hline III & 7 \\
\hline IV & 11 \\
\hline V & 13 \\
\hline \multicolumn{2}{|l|}{ ASA class } \\
\hline I & 1 \\
\hline II & 11 \\
\hline III & 19 \\
\hline \multicolumn{2}{|l|}{ Koval's grade by pre-fracture } \\
\hline I & 12 \\
\hline II & 8 \\
\hline III & 2 \\
\hline IV & 5 \\
\hline V & 3 \\
\hline
\end{tabular}

ASA, American Society of Anesthesiologist

Table 2 In-hospital record of patient underwent primary hemi-arthroplasty 


\begin{tabular}{ll}
\hline In-hospital Record & \\
\hline Intraoperative blood loss (mL) & $200.1 \pm 70.2$ (range, 150-400) \\
Hb of POD1 (g/L) & $82.1 \pm 11.2$ (range, 70-109) \\
Length of stay (days) & $14.3 \pm 3.3$ (range, 10-21) \\
Blood transfusion & $3(9.7 \%)$ \\
$\quad$ Number (\%) & $2.0 \pm 0.7$ (range, 1.5-3) \\
Volume (units) & \\
\hline
\end{tabular}

Hb: Hemoglobulin; POD1: Postoperative Day 1

Figures 


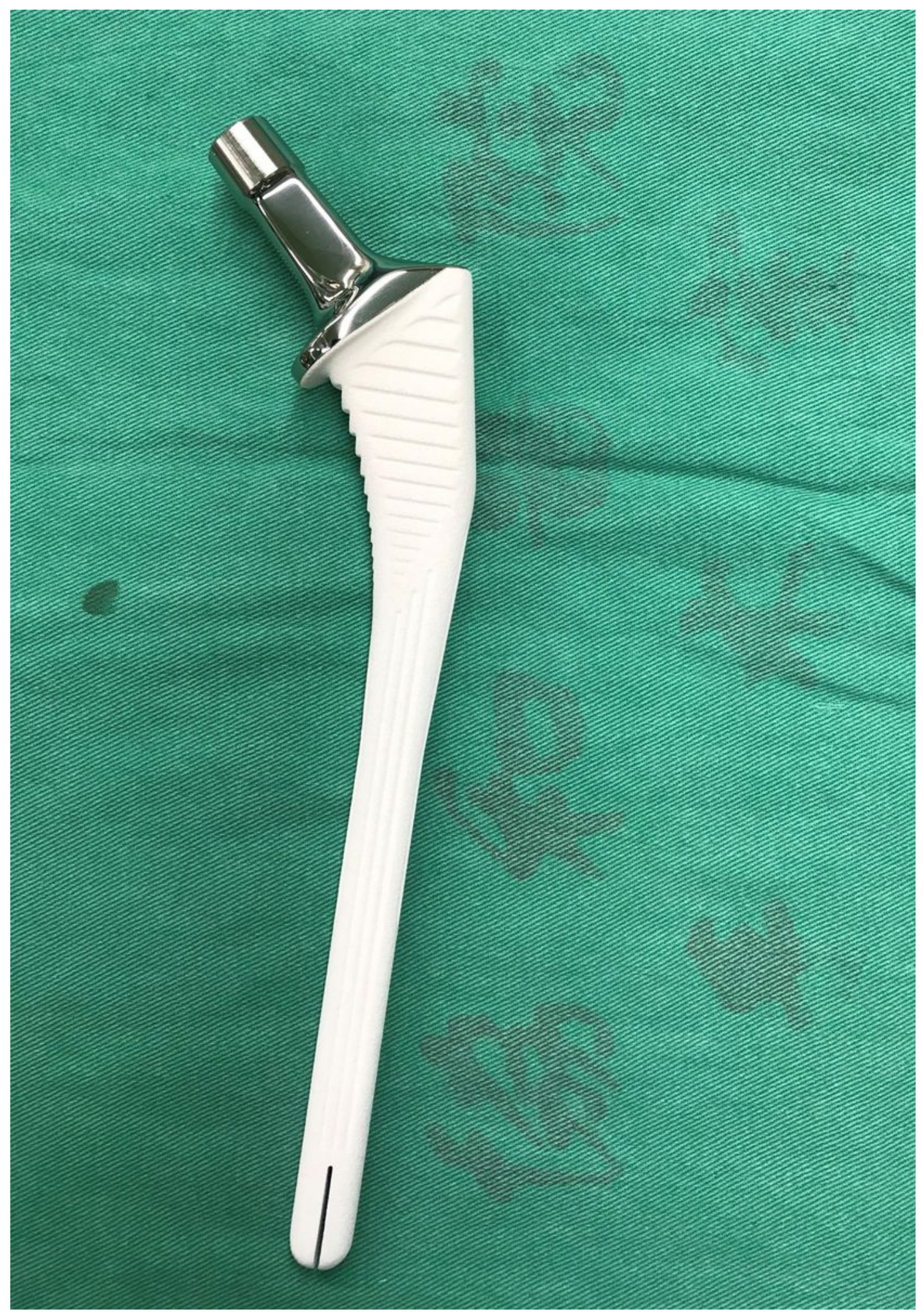

\section{Figure 1}

The bone conserving revision stem is a cementless, tapered titanium stem with a collar and extensive hydroxyapatite coating on the surface. 


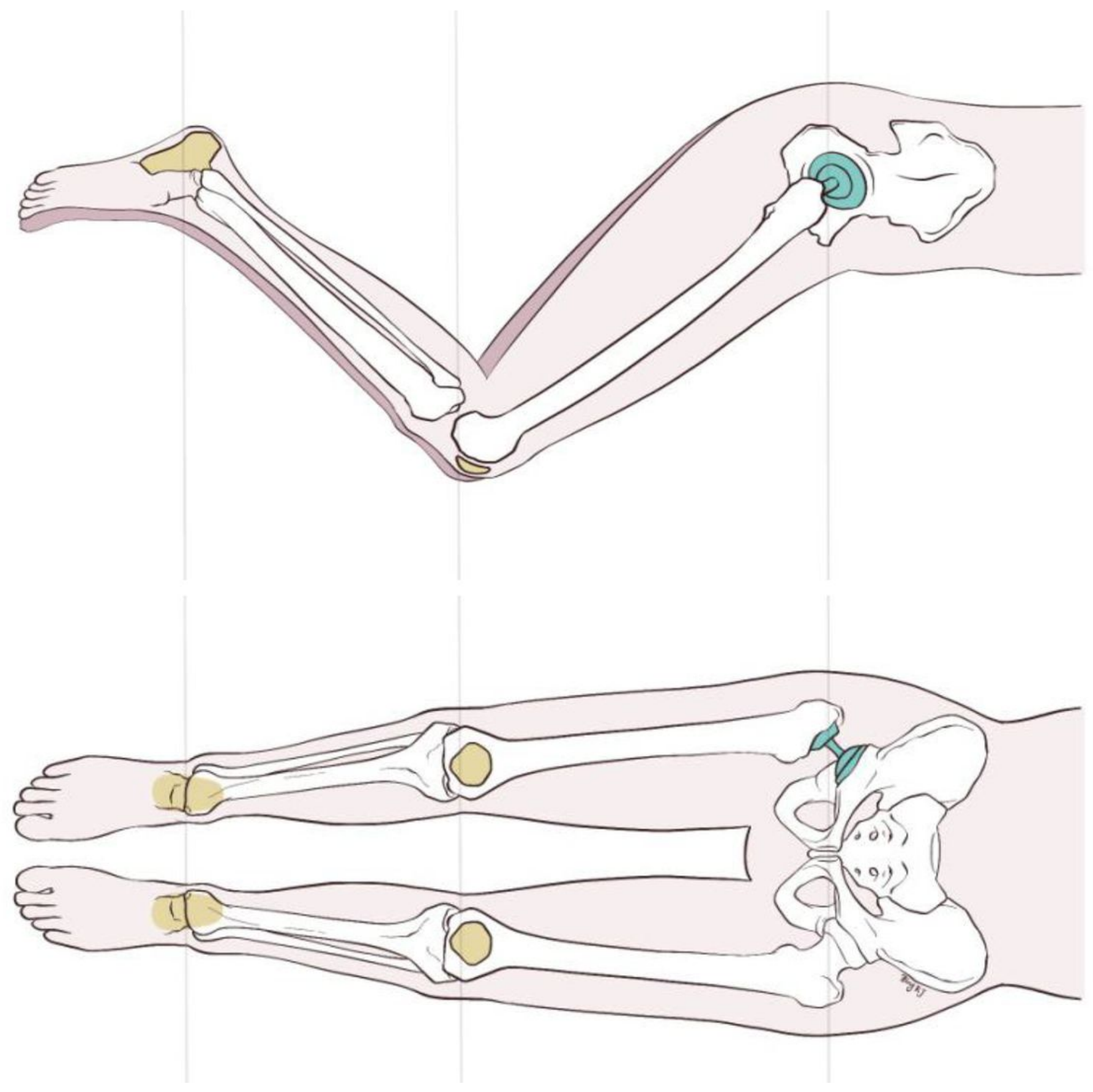

Figure 2

Intra-operative adjustment of lower limb length. Except for intra-operative C-arm imaging, intra-operative adjustment of lower limb length was double-checked by palpation. The patient's pelvis was shifted to a vertical position by palpating and placing both iliac crests at the same vertical plane. The operated leg was placed on top of the contralateral leg with similar abduction angle, and both heels were placed at the same level over the surgical drape. Then, we palpated the two poles of the patella to ensure lower limb length. 


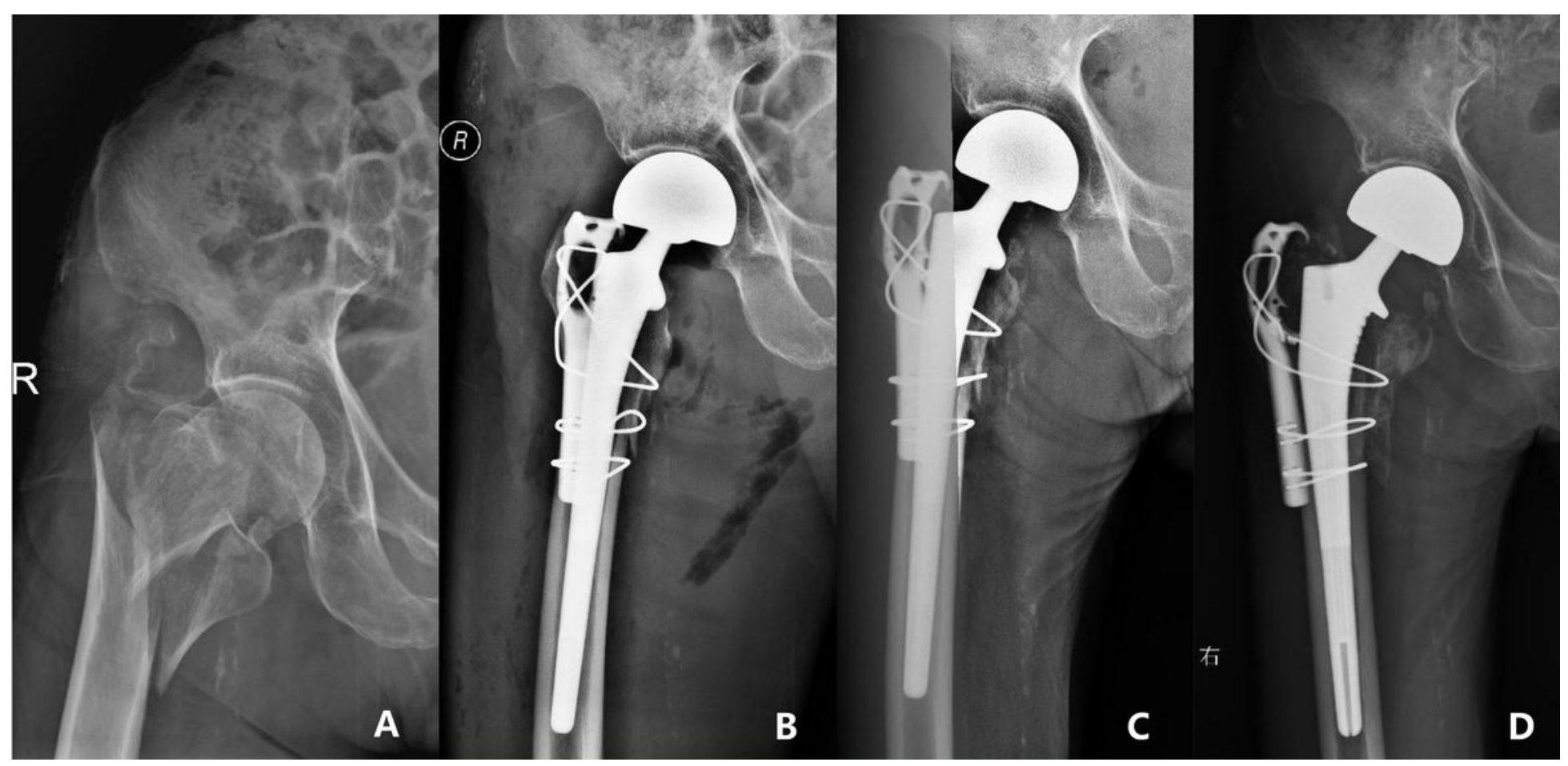

Figure 3

Radiographic illustrations of an 87-year-old (at the time of the surgery) male patient. A: Pre-operative Xray indicated unstable intertrochanteric fracture and osteoporosis; B: Postoperative X-ray of the patient 2 day after the operation; C: Follow-up X-ray of the patient at 3 months after the operation; D: Follow-up Xray of the patient at 18 months after the operation.

\section{Supplementary Files}

This is a list of supplementary files associated with this preprint. Click to download.

- Coverletter.docx 